\title{
Is bronchoscopic lung biopsy helpful in the management of patients with diffuse lung disease?
}

\section{To the Editors:}

We enjoyed reading the recent retrospective analysis by ENSMINGER and PRAKASH [1] of fluoroscopy-guided bronchoscopic lung biopsies (BLB) in patients with suspected diffuse lung disease, which concluded that BLB is a clinically useful test in $\sim 75 \%$ of procedures.

One of the most common indicators for BLB is suspected pulmonary sarcoidosis. In these patients, endobronchial biopsies (EBB) of visible mucosa, in addition to BLB, can be helpful in achieving a diagnosis, even if the mucosa appears macroscopically "normal." Indeed, some studies from the USA, which included patients of African-American descent, reported diagnostic EBB rates of up to $85 \%$, and, if performed in addition to BLB, increased the diagnostic yield by $21 \%[2,3]$ Our population, in the North-East of Scotland, is predominantly white European, with typically milder endobronchial sarcoidosis. Therefore, we hypothesised that EBB, in addition to BLB, would not be as useful in adding to the diagnostic yield in our patient population.

We reviewed case notes of 54 patients with biopsy proven sarcoidosis, recording data including ethnicity, pulmonary function, chest radiograph staging, biopsy method (BLB $\pm E B B)$, endobronchial mucosal appearance and procedural complications [4]. Patients were all white Europeans, with a mean age of 45 yrs. Mean forced vital capacity was $88 \%$ predicted, and the majority of patients had stage II/III disease. All patients had both BLB and EBB. Diagnostic yields from BLB and EBB were 88 and $54 \%$ respectively. Additional EBB increased diagnostic yield by only $6 \%$. Diagnosis with EBB was significantly associated with an "abnormal" endobronchial mucosa $(\mathrm{p}=0.009)$ with a positive predictive value of $71 \%$. The diagnostic yield from EBB of normal appearing mucosa was $27 \%$. In total six patients sustained BLB associated pneumothorax.
In conclusion, our data reinforces the view that bronchoscopic lung biopsies are an extremely useful test in the assessment of patients with suspected diffuse lung disease, notably pulmonary sarcoidosis. Furthermore, while additional endobronchial biopsies may not add much to the overall diagnostic yield in white European patients, it is a simple and safe procedure to perform and may confirm the diagnosis in those patients in which bronchoscopic lung biopsy has failed to yield sufficient lung parenchyma for meaningful histological analysis.

\section{R. MacJannette*, J. Fiddes, K. Kerr ${ }^{\#}$ and O. Dempsey*} Depts of *Respiratory Medicine and "Pathology, Aberdeen Royal Infirmary, Foresterhill, Aberdeen, UK.

\section{STATEMENT OF INTEREST}

None declared.

\section{REFERENCES}

1 Ensminger SA, Prakash UBS. Is bronchoscopic lung biopsy helpful in the management of patients with diffuse lung disease? Eur Respir J 2006; 28: 1081-1084.

2 Shorr AF, Torrington KG, Hnatiuk OW. Endobronchial biopsy for sarcoidosis: a prospective study. Chest 2001; 120: 109-114.

3 Torrington KG, Shorr AF, Parker JW. Endobronchial disease and racial differences in pulmonary sarcoidosis. Chest 1997; 111: 619-622.

4 MacJannette RI, Fiddes JGF, Kerr KM, Dempsey OJ. Should endobronchial biopsies be performed in Caucasian patients with suspected pulmonary sarcoidosis? Eur Respir J 2006; 28 : Suppl. 50, 356S.

\section{Recommendations on the use of exercise testing in clinical practice}

\section{To the Editors:}

Congratulations to the European Respiratory Society (ERS) Task Force for updating their classic report on Clinical Exercise Testing [1] on its 10th anniversary. The original report [2] was the first to summarise the collective views on exercise testing of workers in the respiratory field, and the update provides an opportunity for reinterpretation in light of developments since 1997.

These new developments have included: 1) the emergence of evidence that cycle ergometry may not reproduce the respiratory symptoms of chest patients who are not cyclists; 2) an increased interest in the contribution of pattern of 
breathing to exercise limitation; and 3) a growing impression that ergometry may be beyond the competence of some lung function laboratories. The latter might have contributed to the paucity of material on exercise that was presented at the 2006 ERS Annual Congress.

We are concerned that these and related topics are not addressed adequately in the report, and so make additional suggestions for consideration by the Task Force.

Exercise capacity contributes to the management of both respiratory and cardiac patients and its assessment is an essential component of cardiopulmonary exercise testing [3]. However, whilst the equipment, some protocols and a need to identify the affected bodily systems are shared, the requirements of the two disciplines subsequently diverge. Cardiologists rightly focus on indices of cardiac and circulatory function. In the case of exercise there are rigid guidelines and these give the technicians control of the tests. Respiratory physicians can usefully focus on how lungs that are damaged by disease adapt to the challenge of exercise and how the response can be improved. The circumstances of the test are often pragmatic with the patient in control, so the conduct of the test requires a flexible approach; respiratory technicians adjust to this, but some cardiac technicians feel insecure. Thus, the differences between the two types of assessment operate at a number of levels and for optimal results they should be borne in mind, not ignored. The respiratory dimension, which includes daily living as well as exercise limitation, is the remit of "respiratory exercise testing" and we commend this term in preference to "clinical exercise testing", which glosses over the differences; the latter term is, in this context, essentially nonspecific and has implications for testing disorders outside the thorax.

Apart from for exercise-induced bronchoconstriction there has been no change to the previous recommendation of the ERS and American Thoracic Society that cycle ergometry is the method of choice [2, 3]. This mode of exercise has the advantage that the patient is seated, so is accessible and feels secure. The patient can usually perform what is required and the method lends itself to a progressive protocol. In addition, the test occupies a minimum amount of space in the laboratory and is relatively cheap. The case against cycle ergometry is that respiratory patients usually experience breathlessness during walking or mounting stairs, not cycling, which few patients undertake and which uses different muscles. At the end of a symptom-limited cycling test a patient who experiences breathlessness during daily living may give up on account of fatigue [4]; this has somewhat different features. As a result, the score for breathlessness during daily living cannot be predicted from that obtained on a cycle ergometer [5]. This is despite cycling, on average, being associated with a relatively shallow, more rapid pattern of breathing than walking $[6,7]$; such a pattern increases the ventilation minute volume $[8,9]$ so could predispose to breathlessness. Exercise training on a cycle ergometer might be expected to improve performance during cycling, but not necessarily the exercise capacity and quality of life during daily living.

The differences between the responses to cycling and walking do not appear to be in dispute, and have led some laboratories to replace ergometry with timed walking or shuttle tests. Such tests are fine for assessing exercise capacity, but not for exploring mechanisms. For this, the exercise should normally be carried out on a treadmill using a progressive protocol [10, 11] with the end-point determined by the patient (symptom limited). However, a cycle ergometer should be available for those few patients who find a treadmill daunting. The minimal information is of ventilation, including breathing frequency and tidal volume (pattern of breathing), gas exchange, electrocardiography, body muscle and fat and pulse oximetry. The current additional procedures for assessing dynamic hyperinflation [1] may be more appropriate for specialist laboratories. However, hyperinflation during the course of a progressive symptom limited test reduces the ability to inspire and hence the tidal volume. This reduction is usually apparent on a graph relating ventilation to tidal volume $[12,13]$; such a change might provide sufficient information for routine clinical purposes and this possibility merits investigation.

Breathlessness on exertion during daily living and the associated quality of life are related more closely to an increase in the ventilatory cost of exercise than to impaired lung function [14]. This ventilatory burden can be represented by the ventilation at an oxygen uptake of $1.0 \mathrm{~L} \cdot \mathrm{min}^{-1}\left(45 \mathrm{mmol} \cdot \mathrm{min}^{-1}\right.$; designated $\left.V^{\prime} \mathrm{E}, \mathrm{st}\right)$ which is the level needed for many daily tasks, including the ability to undertake light work [15]. In healthy subjects, the average $V^{\prime} \mathrm{E}$,st is $\sim 23-25 \mathrm{~L} \cdot \mathrm{min}^{-1}$, but in respiratory patients values $>40 \mathrm{~L} \cdot \mathrm{min}^{-1}$ are not uncommon; this high ventilatory burden can only be identified by an exercise test. Examples of conditions where high levels may occur are given in the ERS recommendations [1]. The differential diagnosis is functional breathing, but in our experience differentiation is possible using the results of an exercise test $[16,17]$. The report states that an exercise test cannot uniquely affect diagnosis, but this is possibly too modest; in our view the exploration of such cases should be within the scope of a routine lung function laboratory. In chronic obstructive pulmonary disease the case for respiratory exercise testing is well established [1] and, since the condition is ubiquitous, the exercise assessment should again be conducted at the district level.

In summary, the mechanisms that underlie an increase in ventilation during daily living can be important for diagnosis and clinical management. They are investigated by what is appropriately described as respiratory exercise testing. This differs from cardio-respiratory exercise testing and clinical exercise testing in that the operators focus both mentally and practically on the respiratory consequences of lung diseases. The assessments should preferably be performed using a treadmill, not a cycle ergometer, and, for clinical purposes, be undertaken at the nearest convenient lung function laboratory. Neglect of these aspects detracts from the report of the Task Force: the recommendations should be updated further.

\section{J.E. Cotes and J.W. Reed}

Institute of Molecular and Cell Biology, Medical School, Newcastle upon Tyne, UK.

\section{REFERENCES}

1 Palange $\mathrm{P}$, Ward SA, Carlsen K-H, et al. Recommendations on the use of exercise testing in clinical practice. Eur Respir J 2007; 29: 185-209. 
2 Clinical exercise testing with reference to lung diseases. indications, standardization and interpretation strategies. ERS Task Force on Standardization of Clinical Exercise Testing. European Respiratory Society. Eur Respir J 1997; 10: 2662-2689.

3 American Thoracic Society/American College of Chest Physicians. ATS/ACCP Statement on Cardiopulmonary Exercise Testing. Am J Respir Crit Care Med 2003; 167: 211277.

4 Man WD, Soliman MG, Gearing J, et al. Symptoms and quadriceps fatigue after walking and cycling in chronic obstructive pulmonary disease. Am J Respir Crit Care Med 2003; 168: 562-567.

5 Oga T, Nishimura K, Tsukino M, et al. Dyspnoea with activities of daily living versus peak dyspnoea during exercise in male patients with COPD. Respir Med 2006; 100: 965-971.

6 Thorsen E. Exercise mode affects the relationship between tidal volume and ventilation. Modelling of the response. Eur Respir J 1994; 7: Suppl. 18, 198s.

7 Elliott C. The effects of externally applied breathing resistances. PhD Thesis. University of Newcastle upon Tyne, Newcastle upon Tyne, UK, 1998.

8 Otis AB. The work of breathing. In: Fenn WO, Rahn H, eds. American Physiological Society Handbook of Physiology. Section 3: Respiration. Washington, American Physiological Society, 1964; pp. 463-476.

9 Cotes JE, Reed JW. A new model for describing ventilation during submaximal exercise in healthy men and men with chronic lung disease. Proc Physiol Soc 2006; 3: C39.

10 Porszasz J, Casaburi R, Somfay A, et al. A treadmill ramp protocol using simultaneous changes in speed and grade. Med Sci Sports Exerc 2003; 35: 1596-1603.

11 Cotes JE, Chinn DJ, Miller MR. Exercise testing and interpretation, including reference values. In: Cotes JE, Chinn DJ, Miller MR, eds. Lung Function: Physiology, Measurement and Application in Medicine. 6th Edn. Oxford, Blackwell Publications, 2006; p. 422.

12 Cotes JE, Chinn DJ, Miller MR. Exercise testing and interpretation, including reference values. In: Cotes JE, Chinn DJ, Miller MR, eds. Lung Function: Physiology, Measurement and Application in Medicine. 6th Edn. Oxford, Blackwell Publications, 2006; p. 431.

13 Cotes JE, Johnson GR, McDonald A. Breathing frequency and tidal volume: relationship to breathlessness. In: Porter R, ed. Breathing: Hering-Breuer Centenary Symposium. London, Churchill, 1970; pp. 297-314.

14 Cotes JE, Zejda J, King B. Lung function impairment as a guide to exercise limitation in work-related lung disorders. Am J Respir Crit Care Med 1988; 137: 1089-1093.

15 American Thoracic Society. Evaluation of impairment/ disability secondary to respiratory disorders. Am Rev Respir Dis 1986; 133: 1205-1209.

16 Cotes JE, Reed JW. Disproportionate shallow breathing during exercise as cause of respiratory disability. Eur Respir J 2006; 28: Suppl. 50, 464s.

17 Cotes JE, Reed JW. Exercise ventilation in diffuse pleural disease. Thorax, 2006; 61: Suppl. 11, ii129.

DOI: $10.1183 / 09031936.00006607$

\section{From the authors:}

The members of the European Respiratory Society Task Force on Exercise Testing in Clinical Practice have read with interest the letter from J.E. Cotes and J.W. Reed and are of the opinion that any response to the points raised therein should be placed in the context of the purpose of a recently published Task Force [1]. As stated in the introduction of this Task Force [1]: "The purpose of this document is to present recommendations on the clinical use of exercise testing in patients with cardiopulmonary disease, with particular emphasis on the evidence base for functional evaluation, prognosis and assessment of interventions. While the scope of the document is broad, consideration will focus only on those indices which have demonstrable predictive power". The key phrase here is "evidence base", which represents a clear departure from the objectives of the 1997 Task Force [2]. In recent years, there has been an accumulating body of evidence across a broad range of chronic lung and heart diseases (e.g. chronic obstructive pulmonary disease (COPD), interstitial lung disease (ILD), pulmonary vascular disease and chronic heart failure (CHF)) from studies using cycle ergometer protocols and field tests. It is upon this collective evidence base that the Task Force has formulated its recommendations for exercise testing in clinical practice.

Furthermore, the Task Force was written with the practicing clinician in mind, to raise awareness of the additional value of measuring exercise tolerance in clinical practice. That is, to "allow resolution of practical issues that often confront the clinician, such as: 1) "When should an evaluation of exercise intolerance be sought?"; 2) "Which particular form of test should be asked for?"; and 3) "What cluster of variables should be selected when evaluating prognosis for a particular disease or the effect of a particular intervention?" [1]". Therefore, it was expressly intended not to provide a high level of technical detail with respect to the design, implementation and interpretation of a cardiopulmonary exercise test (CPET) or a field-based walking test; other documents have done this successfully in the past [2,3].

We believe that the Task Force report has addressed several of the "new developments" that have been highlighted by J.E. Cotes and J.W. Reed. These include: 1) the differences in patterns of physiological response between cycle ergometer exercise and walking that have been observed in COPD; 2 ) the influence of lung dynamic hyperinflation on dyspnoeic sensation and exercise intolerance, not only in pulmonary disease but also in heart disease; 3 ) the impact of particular exercise-based indices in prognostic evaluation, such as the slope of the minute ventilation $\left(V^{\prime} \mathrm{E}\right)$-carbon dioxide output $\left(V^{\prime} \mathrm{CO}_{2}\right)$ relationship and the ventilatory equivalent for carbon dioxide $\left(V^{\prime} \mathrm{E} / V^{\prime} \mathrm{CO}_{2}\right)$ at the lactate threshold during incremental cycle ergometry in CHF patients, and arterial oxygen desaturation during walking tests in patients with ILD; and 4) the utility of high-intensity, constant work-rate "endurance" cycle ergometer protocols for assessing the effects of interventions

Regarding the recommendation that J.E. Cotes and J.W. Reed make concerning terminology, we suggest that there are generic aspects of exercise-test design and analysis that should be common to evaluation of the pulmonary and cardiac patient. The use to which these are subsequently put is, of 